NASA Contractor Report 178218

ICASE REPORT NO. $86-52$

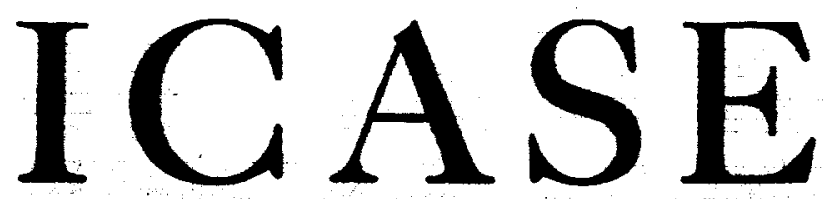

(NASA-CR-1782 18) NAVE BNVELCEE TECHNIQUE FOR WOLTIMODE IIVE GUIDE FECBLELS (MASA) $22 \mathrm{p}$

CSCL 12A

พ $87-18318$

Unclas

$63 / 59 \quad 43387$

WAVE ENVELOPE TECHNIQUE FOR MULTIMODE WAVE

GUIDE PROBLEMS

S. I. Hariharan

S. I. Sudharsanan

NASA Contracts No. NAS1-17070, NAS1-18107

December 1986

INSTITUTE FOR COMPUTER APPLICATIONS IN SCIENCE AND ERGINEERING

NASA Langley Research Center, Hampton, Virginia 23665

Operated by the Universities Space Research Association

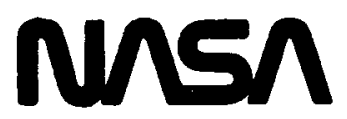

National Aeronautics and

Space Administration

Lengley Research Center

Hamplon, Virginia 23665 


\title{
Wave Envelope Technique for Multimode Wave Guide Problems
}

\author{
S.I.Hariharan \\ Department of Mathematical Sciences \\ University of Akron, Akron, OH 44325 \\ and \\ Institute for Computer Applications in Science and Engineering \\ S.I.Sudharsanan \\ University of Tennessee Space Institute, Tullahoma, TN 37388
}

\begin{abstract}
In this paper we propose a fast method for solving wave guide problems. In particular, we consider the guide to be inhomogeneous and allow propagation of waves of higher order modes. Such problems have been handled successfully for acoustic wave propagation problems with single mode and fin ite length. This paper extends this concept to electromagnetic wave guides with several modes and infinite length. The method is described and results of computations are presented.
\end{abstract}

Research was supported by the National Aeronautics and Space Administration under NASA Contract Nos. NAS1-17070 and NAS1-18107 while the first author was in residence at ICASE, NASA Langley Research Center, Ham pton, VA 23665-5225, and by NASA Grant No. NAG-1-624. 


\title{
Wave Envelope Technique for Multimode Wave Guide Problems
}

\author{
S.I.Hariharan \\ Department of Mathematical Sciences \\ University of Akron, Akron, $\mathrm{OH} 44325$ \\ and \\ Institute for Computer Applications in Science and Engineering

\section{S.I.Sudharsanan} \\ University of Tennessee Space Institute, Tullahoma, TN 37388
}

\section{Introduction}

This paper presents a method to accelerate computations involving electromagnetic wave guide problems in two dimensions. The wave guide can be finite in length or infinitely long and can have a varying index of refraction. The emphasis here is on guides with infinite length because they are difficult to handle. In [4] Kriegsmann describes a similar situation and we shall make comparisons with his results. However, the method is applicable to more general wave guide problems. For example, acoustic wave guide problems (which are treated by Baumeister in [1] and [2]) and optics problems involving guided waves such as fiber optics can be treated by this method. Though the discussion here pertains to two dimensions, the method could easily be extended to three-dimensional problems with cylindrical symmetry. Another advantage of the method is it can be implemented on a personal computer.

The tool we use here is a combination of two ideas. The general wave guide problems are posed in the frequency domain, i.e., all associated fields have a single angular frequency $\omega$ and have the time 
dependency of the form $e^{i \omega x}$. The first idea is to solve the corresponding time dependent problem using the "limiting Amplitude Principle." The limiting amplitude principle states that the solution of a hyperbolic partial differential equation (which is the case here), with a periodic forcing function, will approach a time periodic solution with the same period as $t \rightarrow \infty$. This is a known concept; however, a rigorous justification in general, including our case, is a difficult task. We refer readers to the paper of Morawetz [5] for technical details. Even though the time dependent problem is much harder to treat, it has its own advantage. The associated wave equation, which is weakly elliptic, possesses interior resonant values for which there are no unique solutions. It is usually difficult to locate such resonant frequencies. Considering the problem in the time domain conveniently avoids the situation, and the solution passes through these resonant values smoothly yielding the desired solution. This is the advantage of the limiting amplitude principle.

The next concept we need here is the wave envelope method. The basis of the method heavily relies on the following fact: to compute solutions of time harmonic waves, one usually requires fincr meshes as the frequency becomes larger and the corresponding computational time increases. However, the wave contain s an envelope which is the amplitude. Usually, the calculation of the amplitude is the goal. For example, in acoustic problems, the root mean square ( r.m.s. ) value of the pressure is required rather than the actual time history of the wave. Thus, instead of resolving the actual wave oscillations, one may consider a coarse mesh on the envelope as an alternative procedure. A typical situation is depicted in figure 1.1. This procedure turns out to be closely connected to the geometric optics principle.

Let us introduce problem under consideration. The problem is posed in the frequency domain as follows: Determine u $\varepsilon C^{2}(\Omega)$, such that

$$
\left(P_{F}\right)\left\{\begin{array}{l}
\Delta u+k^{2} n^{2}(x, y) u=0 \text { in } \Omega \\
u(x, 0)=0 \\
u(x, 1)=0 \\
u(x, y) \approx e^{i k_{1} x} \sin \pi y \text { as } x \rightarrow-\infty, \quad\left(k_{1}=\sqrt{k^{2}-\pi^{2}}\right), \\
u \text { satisfies a radiation condition as } x \rightarrow-\infty,
\end{array}\right.
$$


where,

$$
\mathrm{n}(\mathrm{x}, \mathrm{y})= \begin{cases}\mu(\mathrm{x}, \mathrm{y}) & \text { in } \mathbf{R} \\ 1 & \text { in } \mathbf{R}_{\mathrm{c}}\end{cases}
$$

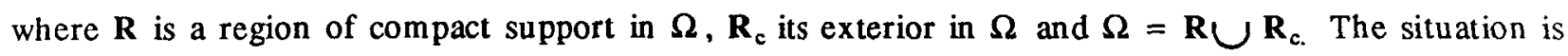
illustrated in Figure 1.2.

The physical process here says there is a wave incident in the guide $(x \rightarrow-\infty)$ of magnitude $e^{i k_{1} x} \sin \pi y$ which impinges through a region $\mathbf{R}$ of varying index of refraction and decays at $\infty$. Problem $\left(P_{F}\right)$ can be derived from Maxwell's equations for transverse magnetic field with $u$ being the potential of the electric field (see [3]). The homogeneous Dirichlet boundary condition on $y=0$ and $y=1$ indicate the guide is insulated.

Solving $\left(\mathrm{P}_{\mathrm{F}}\right)$ is the goal of this paper. As we mentioned earlier, first we shall consider the problem as a time-dependent problem. The boundary conditions that simulate the behavior at $+\infty$ and $-\infty$ at finite distances will be considered. Then we will convert into a fast mode with wave envelope technique. The discretization will then be considered. Finally, numerical examples will be presented.

\section{The Time-Dependent Problem}

We consider the time-dependent problem corresponding to $\mathrm{P}_{\mathrm{F}}$ :

$$
\left(P_{T}\right)\left\{\begin{array}{l}
\Delta \hat{\mathbf{u}}-\mathrm{n}^{2}(\mathrm{x}, \mathrm{y}) \hat{\mathrm{u}}_{\mathfrak{t}}=0 \\
\hat{\mathbf{u}}(\mathrm{x}, 0, \mathrm{t})=0 \\
\hat{\mathbf{u}}(\mathrm{x}, 1, \mathrm{t})=0 \\
\hat{\mathbf{u}}(\mathrm{x}, \mathrm{y}, \mathrm{t}) \approx \mathrm{e}^{+\mathrm{ik}_{1^{\mathrm{x}}-\mathrm{ik} t}} \sin \pi \mathrm{y}, \text { as } \mathrm{x} \rightarrow-\infty \\
\hat{\mathbf{u}} \text { satisfies a radiation condition. }
\end{array}\right.
$$

The goal is to solve for the time-dependent variable $\hat{u}$ from a state of rest. According to the limiting amplitude principle, $\hat{u}(x, y, t)$ should become $u(x, y) e^{-i k t}$ as $t \rightarrow \infty$. A sample calculation to show that the time harmonic steady state is achieved is shown in Figure $1.3(a, b, c, d)$. In this figure, the time history of the real part of the electric field is shown over the indicated span of time. Around $t=20$, the harmonic steady state is achieved. For illustrative purposes and also to show the stability of the solution procedure, the results are indicated up to a time $t=50$. The structure of the solution remain $s$ 
unch anged.

So far we have not specified the boundary condition required as $x \rightarrow \infty$. This was omitted because we need boundary conditions at finite distances for computational purposes anyway. Both the inflow conditions and the radiations condition must be specified at finite distances and should indicate the behavior at infinity. We consider the truncated region $\bar{\Omega}$ given as a rectangle $\bar{\Omega}=[-L, L] \times[0,1]$, where $L$ is typically large (Figure 1.4).

For $L$ large, the governing equation of $\left(P_{T}\right)$ has constant coefficients (i.e., $\left.n(x, y)=1\right)$. Thus, near $x=-L$ and $x=L$ the solution using separation of variables may be written as

$$
\begin{aligned}
& \hat{u}(x, y, t)=e^{i k_{1} x-i k t} \sin \pi y+\sum_{n=1}^{\infty} R_{n} e^{-i k_{n} x-i k t} \sin n \pi y \\
& \hat{u}(x, y, t)=\sum_{n=1}^{\infty} T_{n} e^{+i k_{n} x-i k t} \sin n \pi y .
\end{aligned}
$$

Here

$$
k_{n}=\sqrt{k^{2}-n^{2} \pi^{2}}
$$

and $R_{n}$ and $T_{n}$ are identified as the reflection ans transmission coefficients, respectively. For propagating waves, $k^{2}-n^{2} \pi^{2}>0$. For a given $k$, the largest number $n$ satisfying the in equality is called the 'mode'. For example, for $\mathbf{k}=3.5$ we see that $\mathbf{n}=1$ and we say that there is one propagating mode. In such a case $k_{i},(i=2,3, \ldots)$ become purely imaginary, and the wave components corresponding to the coefficients $R_{i}$ and $T_{i}(i=2,3, \ldots)$ will decay. They are called evanescent waves.

For the relation (2.3) we see that for $\pi<k<2 \pi$ there will be one mode propagating in the guide. Also the solution near $x=-L$ and $x=L$ will be dominated by $R_{1}$ and $T_{1}$. In fact, we can write (2.1) and (2.2) in an approximate sense

$$
\begin{array}{ll}
\hat{u}(x, y, t)=\left(e^{i k_{1} x}+R_{1} e^{-i k_{1} x}\right) e^{-i k t} \sin \pi y, & (\text { near } x=-L), \\
\hat{u}(x, y, t)=T_{1} e^{i k_{1} x-i k t} \sin \pi y, & (\text { near } x=L) .
\end{array}
$$

To prescribe (2.4) and (2.5) as boundary conditions at inflow $(x=-L)$ and outflow $(x=L)$, we need to know $R_{1}$ and $T_{1}$ apriori. This information is not available since this is part of the solution. On the other hand, we can annihilate $R_{1}$ and $T_{1}$ using appropriate differential operators. The net result is 


$$
\begin{aligned}
B_{1} \hat{\mathbf{u}} \equiv \hat{\mathbf{u}}_{\mathrm{x}}-\frac{\mathbf{k}_{1}}{\mathbf{k}} \hat{\mathbf{u}}_{\mathrm{t}}=2 \mathrm{ik}_{1} \mathrm{e}^{\mathrm{i \mathbf {k } _ { 1 } x - i \mathbf { k t }} \sin \pi \mathrm{y},} & (\text { at } \mathrm{x}=-\mathrm{L}), \\
\mathrm{B}_{2} \hat{\mathbf{u}} \equiv \hat{\mathbf{u}}_{\mathrm{x}}+\frac{\mathbf{k}_{1}}{\mathbf{k}} \hat{\mathbf{u}}_{\mathfrak{t}}=0, & (\text { at } \mathrm{x}=\mathrm{L}) .
\end{aligned}
$$

Thus, (2.6) and (2.7) may be taken as the approximate boundary conditions at the inflow and outflow respectively.

For frequencies in the range $\pi<k<4 \pi$, we see from $(2.1),(2.2)$, and (2.3) that the dominance of $R_{2}$ and $T_{2}$ become important. The procedure can be extended to this case by retaining one more term in the expansions of (2.1) and (2.2). Thus, in the approximate sense

$$
\begin{array}{ll}
\hat{u}(x, y, t)=\left(e^{i k_{1} x} \sin \pi y+R_{1} e^{-i k_{1} x} \sin \pi y+R_{2} e^{-i k_{2} x} \sin 2 \pi y\right) e^{-i k t} & (\text { near } x=-L) \\
\hat{u}(x, y, t)=\left(T_{1} e^{i k_{1} x} \sin \pi y+T_{2} e^{i k_{2} x} \sin 2 \pi y\right) e^{-i k t} & (\text { near } x=L)
\end{array}
$$

Analogous to (2.6) and (2.7) we obtain second-order boundary operators as follows:

$$
\begin{array}{ll}
{\left[\frac{\partial}{\partial x}-\frac{\mathbf{k}_{2}}{\mathbf{k}} \frac{\partial}{\partial t}\right]\left[\frac{\partial}{\partial x}-\frac{\mathbf{k}_{1}}{\mathbf{k}} \frac{\partial}{\partial t}\right] \hat{\mathbf{u}}=2 \mathbf{k}_{1}\left(\mathbf{k}_{1}-\mathbf{k}_{2}\right) \mathrm{e}^{i \mathbf{k}_{1} \mathrm{x}-\mathrm{ikt},},} & (\mathrm{x}=-\mathrm{L}), \\
{\left[\frac{\partial}{\partial \mathrm{x}}+\frac{\mathbf{k}_{2}}{\mathbf{k}} \frac{\partial}{\partial t}\right]\left[\frac{\partial}{\partial \mathrm{x}}+\frac{\mathbf{k}_{1}}{\mathrm{k}} \frac{\partial}{\partial t}\right] \hat{\mathbf{u}}=0,} & (\mathrm{x}=+\mathrm{L}) .
\end{array}
$$

For higher values of $\mathbf{k}$, this procedure may be repeated to obtain high-order boundary conditions. Details will be found in Kriegsmann [4]. While this procedure remains elegant in extracting boundary conditions, their stability with respect to the interior equation is difficult to examine. In fact, the boundary conditions arising from (2.10) and (2.11) were unstable in our numerical calculations, but reasonably accurate results were obtained with the lower order boundary conditions (2.6) and (2.7). Th is is in contrast to the results that are reported in Kriegsmann [4].

\section{Wave Envelope Technique}

Here we want to make a further change in the dependent variable $\hat{u}$ of $\left(\mathrm{P}_{T}\right)$ so that the new variable will not oscillate rapidly in the axial direction. For simplicity and to fix ideas, let us consider the one-dimensional wave motion governed by

$$
\mathbf{u}_{\mathfrak{t t}}=\mathbf{u}_{\mathbf{x x}}
$$

Let us introduce a new variable $v(x, t)$ such that 


$$
u(x, t)=e^{\eta x} v(x, t)
$$

Substitution of (3.2) in (3.1) yields

$$
\mathrm{v}_{\mathrm{tz}}=\mathrm{v}_{\mathrm{xx}}+2 \eta \mathrm{v}_{\mathrm{x}}+\eta^{2} \mathrm{v} .
$$

Let us further specialize to the situation of time harmonic fields with a single angular frequency $k$, i.e.,

$$
v(x, t)=e^{-i k t} z(x) .
$$

This yields

$$
z^{\prime \prime}+2 \eta z^{\prime}+\left(\eta^{2}+k^{2}\right) z=0
$$

whose characteristic roots are

$$
\lambda=-\eta \pm \mathbf{i k}
$$

Thus, the general solution is given by

$$
z(x)=e^{-\eta x}\left(A e^{i k x}+B e^{-i k x}\right) .
$$

We further specialize (3.2) to the situation that the wave motion is unidirectional moving from left to right. Then (3.7) becomes

$$
z(x)=A e^{-(\eta-i k) x} .
$$

At this point, the choice of $\eta$ is completely arbitrary. Let $\eta=i \eta^{*}$; then (3.8) further reduces to

$$
z(x)=A e^{-i\left(\eta^{*}-k\right) x}
$$

For $\eta^{*}=\mathbf{k}$, we see that $\mathbf{z}(\mathbf{x})=\mathrm{A}$, which yields the amplitude directly. Basically, we require only two mesh points for such a calculation. Of course, this is not the problem we need to solve. However, the concept is a valuable tool in understanding the method.

Let us repeat this process for a problem with variable index of refraction $n(x)$. Here the wave motion is described by

$$
\mathrm{n}^{2}(\mathrm{x}) \mathrm{u}_{\mathrm{u}}=\mathrm{u}_{\mathrm{xx}} .
$$

The process described by (3.2) to (3.5) with $\eta=$ i $\eta^{*}$ yields

$$
z_{x x}+2 i \eta^{*} z_{x}+\left(n^{2}(x) k^{2}-\eta^{* 2}\right) z=0 .
$$

Let us compare this equation with equation (3.5). In order to capture the amplitude, we set $\mathrm{k}=\eta^{*}$. However, it is no longer feasible in (3.11) to do so, due to the variable nature of $n(x)$. Nevertheless, 
for numerical computations, one may slow down the variations of the wave suggested by (3.9) with a choice of $\eta^{*}$ given by

$$
\eta^{*}=k \max _{0 \leq x \leq L} n(x)
$$

In such a situation, the illustration prescribed in figure 1.1 holds. Perhaps there are other ways to obtain the best choice of $\eta^{*}$ which we do not know at this time. The method is known as the wave envelope method.

Now let us extend this concept to problem $\left(\mathrm{P}_{\mathrm{T}}\right)$ posed in the truncated domain $\bar{\Omega}$ with boundary conditions (2.6) and (2.7). To monitor the solution over a period of length $2 \pi$ in the axial direction, we chose $\eta^{*}=2 \pi \eta^{+}$. We do not make an y choices in the y-direction as the oscillations are rather slow in that direction. Thus, our transformation is

$$
\hat{\mathbf{u}}=\mathrm{e}^{-\mathrm{i} 2 \pi \eta^{+} \mathrm{x}} \mathrm{p}(\mathrm{x}, \mathrm{y}, \mathrm{t}) \text {. }
$$

Substituting this relation in to $\left(\mathrm{P}_{\mathrm{T}}\right)$ with boundary conditions (2.6) and (2.7), we obtain the following problem:

Find $\mathrm{p} \varepsilon \mathrm{C}^{2}(\bar{\Omega})$ such that

$$
\left(P _ { E ) } \left\{\begin{array}{ll}
n^{2}(x, y) p_{\mathfrak{t}}=\Delta p-i 4 \pi \eta^{+} p_{x}-\left(2 \pi \eta^{+}\right)^{2} p, & \text { in } \bar{\Omega} \\
p(x, 0, t)=0 & \\
p(x, 1, t)=0 & (\text { at } x=-L) \\
p_{x}=i 2 \pi \eta^{+} p+\frac{k_{1}}{k} p_{t}+2 i k_{1} e^{i k_{1} x-i k t+2 \pi i \eta^{+} x} \sin \pi y & (\text { at } x=L), \\
p_{x}=i 2 \pi \eta^{+} p-\frac{k_{1}}{k} p_{t} &
\end{array}\right.\right.
$$

which is our wave envelope problem.

Historically, the term inology wave envelope arises because we attempt to resolve the envelope of the wave rather that the actual oscillation of the wave.

\section{Numerical Procedure}

The procedure consists of a finite difference method via a variation of the finite area method. We refer to Baumeister [1], [2] where we obtained guidance in constructing the procedure. The derivation begins from the governing equation for $\left(\mathrm{P}_{\mathrm{E}}\right)$, which is in tegrated over a cell (Figure 4.1) 


$$
\int_{t-\frac{\Delta t}{2}}^{t+\frac{\Delta t}{2}} \int_{-}^{+} \int_{-}^{+}\left[n^{2}(x, y) P_{t t}-\Delta p+i 4 \pi \eta^{+} p_{x}+\left(2 \pi \eta^{+}\right)^{2} p\right] d x d y d t=0
$$

where a plus sign $(+)$ in the upper limit of integration indicates evaluation at either the upper or righthand boundary of the integration of the cell and a minus sign (-) in the lower limit indicates evaluation at the lower or the left-hand boundary of the cell. In this procedure, the integral involving second derivatives in time is approximated by a standard central difference scheme, i.e.,

$$
\int_{t-\frac{\Delta t}{2}}^{t+\frac{\Delta t}{2}} \int_{-}^{+} \int_{-}^{+} \frac{\partial^{2} p}{\partial t^{2}} d x d y d t=\frac{p_{i, j}^{k+1}-2 p_{i, j}^{k}+p_{i, j}^{k-1}}{\Delta t} A
$$

where $\mathrm{A}$ is the area of the cell. For boundary cells 3 and $4, \mathrm{~A}=\frac{\Delta \mathrm{x}}{2} \Delta \mathrm{y}$, for interior cells 1 and 2 , $\mathrm{A}=\Delta \mathrm{x} \Delta \mathrm{y}$

For an interior point the integration is carried out in a straightforward manner. As in [2] we obtain an explicit difference scheme

$$
\begin{gathered}
\mathrm{p}_{\mathrm{i}, \mathrm{j}}^{\mathrm{k}+1}=\left[\frac{\Delta \mathrm{t}}{\Delta \mathrm{yn}}\right]^{2}\left[\left\{\left[\frac{\Delta \mathrm{y}}{\Delta \mathrm{x}}\right]^{2}-\mathrm{i} 2 \pi \eta^{+}\left[\frac{\Delta \mathrm{y}}{\Delta \mathrm{x}}\right]^{2}\right\} \mathrm{p}_{i+1, j}^{\mathrm{k}}\right. \\
+\left\{\left[\frac{\Delta \mathrm{y}}{\Delta \mathrm{x}}\right]^{2}+\mathrm{i} 2 \pi \eta^{+}\left[\frac{\Delta \mathrm{y}}{\Delta \mathrm{x}}\right]^{2}\right\} \mathrm{p}_{i-1, j}^{\mathrm{k}}+\mathrm{p}_{\mathrm{i}, \mathrm{j}+1}^{\mathrm{k}}+\mathrm{p}_{i, j-1}^{\mathrm{k}} \\
\left.+\left\{-\left(2 \pi \eta^{+} \Delta \mathrm{y}\right)^{2}-2-2\left[\frac{\Delta \mathrm{y}}{\Delta \mathrm{x}}\right]^{2}+2\left[\frac{\Delta \mathrm{y}}{\Delta \mathrm{t}} \eta\right]^{2}\right\} \mathrm{p}_{i, j}^{\mathrm{i}}\right] \\
-\mathrm{p}_{i, j}^{\mathrm{k}-1} .
\end{gathered}
$$

The inflow boundary condition, (see $P_{E}$ ) must be imposed correctly. We shall show this procedure. The same procedure holds at the outflow boundary and for other cells. We consider the spatial derivative part of (4.1) at the inflow

$$
\int_{t-\frac{\Delta t}{2}}^{t+\frac{\Delta t}{2}} \int_{-}^{+} \int_{-}^{+}\left[p_{x x}+p_{y y}-i 4 \pi \eta^{+} p_{x}-\left(2 \pi \eta^{+}\right)^{2} p\right] d x d y d t
$$


We shall decompose this into four parts: $I_{1}, I_{2}, I_{3}$, and $I_{4}$ for each integral in the expression

$$
\begin{gathered}
\left.I_{1}=\int_{t-\frac{\Delta t}{2}}^{t+\frac{\Delta t}{2}} \int_{-}^{+} \int_{-}^{+} p_{x x} d x d y d t=\int_{-}^{+} \int_{-}^{+}\left[\frac{\partial p}{\partial x}\right]+-\int \frac{\partial p}{\partial x}\right]-d y d t \\
=\int_{t-\frac{\Delta t}{2}}^{t+\frac{\Delta t}{2}} \int_{-}^{+}\left[\left[\frac{p_{i+1, j}^{k}-p_{i, j}^{k}}{\Delta x}-2 \pi \eta^{+} i_{i, j}^{k}+\frac{k_{1}}{k} \frac{\partial p}{\partial t}\right.\right. \\
\left.\left.+2 i k_{1} e^{i k_{1} x-i k t+2 \pi i \eta^{+} x} \sin \pi y\right]\right] d y d t \\
=\int_{i-\frac{\Delta t}{2}}^{t+\frac{\Delta t}{2}}\left[\left\{\frac{p_{i+1, j}^{k}-p_{i, j}^{k}}{\Delta x}-2 \pi i p_{i, j}^{k}-\frac{k_{1}}{k} \frac{\partial p}{\partial t}\right\} \Delta y\right. \\
\left.+\frac{2 i k_{1}}{\pi} e^{i k_{1} x-i k t+2 \pi i \eta^{+} x}\left\{\cos \left(\pi y_{+}\right)-\cos \left(\pi y_{-}\right)\right\}\right] d t \\
=\left\{p_{i+1, i}^{k}-p_{i, j}^{k}\left(1+2 \pi i \eta^{+} \Delta x\right)\right\} \frac{\Delta y \Delta t}{\Delta x}-\frac{k_{1} \Delta y}{k} \frac{p_{i, j}^{k+1}-p_{i, j}^{k-1}}{2} \\
-\left\{\cos \left(\pi y_{+}\right)-\cos \left(\pi y_{-}\right)\right\} \frac{2 k_{1}}{\pi k} e^{i k_{1} x+2 \pi i \eta^{+} x}\left[e^{-k_{+}}-e^{i k t_{-}}\right]
\end{gathered}
$$

where

$$
\begin{aligned}
& y_{+}=j \Delta y+\frac{1}{2} \Delta y, \\
& y_{-}=j \Delta y-\frac{1}{2} \Delta y, \\
& t_{+}=k \Delta t+\frac{1}{2} \Delta t, \\
& t_{-}=k \Delta t-\frac{1}{2} \Delta t,
\end{aligned}
$$

and

$$
p_{i, j}^{k}=p(i \Delta x, j \Delta y, k \Delta t)
$$

By a similar calculation we obtain

$$
\begin{gathered}
I_{2}=\int_{t-\frac{\Delta t}{2}}^{t+\frac{\Delta t}{2}} \int_{-}^{+} \int_{-}^{+} p_{y y} d x d y d t \\
=\frac{p_{i, j+1}^{k}-2 p_{i, j}^{k}+p_{i, j-1}^{k}}{\Delta y} \frac{\Delta x}{2} \Delta t \\
I_{3}=i 4 \pi \eta^{+} \int_{t-\frac{\Delta t}{2}}^{t+\frac{\Delta t}{2}} \int_{-}^{+} \int_{-}^{+} p_{x} d x d y d t \\
=i 4 \pi \eta^{+} \Delta y \Delta t\left\{\frac{p_{i+1, j}^{k}-p_{i, j}^{k}}{2}\right\}, \\
I_{4}=\left(2 \pi \eta^{+}\right)^{2} \int_{t-\frac{\Delta t}{2}}^{t+\frac{\Delta t}{2}} \int_{-}^{+} \int_{-}^{+} p d x d y d t
\end{gathered}
$$




$$
=\left(2 \pi \eta^{+}\right)^{2} p_{i, j}^{k} \frac{\Delta x}{2} \Delta y \Delta t .
$$

Therefore, (4.4) $=I_{1}+I_{2}-I_{3}-I_{4}$.

Repeating the above calculations in cells 1 through 4, we have

$$
p_{i, j}^{k+1}=a_{m}\left[b_{m} p_{i+1, j}^{k}+c_{m} p_{i-1, j}^{k}+d_{m} p_{i, j+1}^{k}+e_{m} p_{i, j-1}^{k}+f_{m} p_{i, j}^{k}\right]+g_{m} p_{i, j}^{k-1}+h_{m}
$$

Where the coefficients $a_{m}, b_{m}, c_{m}, d_{m}, e_{m}, f_{m}, g_{m}$ and $h_{m}$ are given in the following table:

\begin{tabular}{|l|c|c|c|c|c|c|c|c|}
\hline REGION & $\mathrm{a}_{\mathrm{m}}$ & $\mathrm{b}_{\mathrm{m}}$ & $\mathrm{c}_{\mathrm{m}}$ & $\mathrm{d}_{\mathrm{m}}$ & $\mathrm{e}_{\mathrm{m}}$ & $\mathrm{f}_{\mathrm{m}}$ & $\mathrm{g}_{\mathrm{m}}$ & $\mathrm{h}_{\mathrm{m}}$ \\
\hline GENERAL & $\mathrm{a}_{1}$ & $\mathrm{~b}_{1}$ & $\mathrm{c}_{1}$ & 1.0 & 1.0 & $\mathrm{f}_{1}$ & -1.0 & 0 \\
\hline OBSTACLE & $\mathrm{a}_{2}$ & $\mathrm{~b}_{1}$ & $\mathrm{c}_{1}$ & 1.0 & 1.0 & $\mathrm{f}_{2}$ & -1.0 & 0 \\
\hline INLET & $\mathrm{a}_{3}$ & $\mathrm{~b}_{3}$ & 0 & $\frac{\Delta \mathrm{x}}{2 \Delta \mathrm{y}}$ & $\frac{\Delta \mathrm{x}}{2 \Delta \mathrm{y}}$ & $\mathrm{f}_{3}$ & $\mathrm{~g}_{3}$ & $\mathrm{~h}_{3}$ \\
\hline EXIT & $\mathrm{a}_{3}$ & 0 & $\mathrm{c}_{4}$ & $\frac{\Delta \mathrm{x}}{2 \Delta \mathrm{y}}$ & $\frac{\Delta \mathrm{x}}{2 \Delta \mathrm{y}}$ & $\mathrm{f}_{3}$ & $\mathrm{~g}_{3}$ & 0 \\
\hline
\end{tabular}

and where

$$
\begin{gathered}
a_{1}=\left[\frac{\Delta t}{\Delta y \eta}\right]^{2} \\
a_{2}=\left[\frac{\Delta t}{\Delta y \bar{n}}\right]^{2} \\
a_{3}=\frac{2 \Delta t^{2}}{\Delta y\left[\eta^{2} \Delta x+\frac{k_{1}}{\omega} \Delta t\right]} \\
b_{1}=\left[\frac{\Delta y}{\Delta x}\right]^{2}-2 \pi i \eta^{+} \frac{\Delta y^{2}}{\Delta x} \\
b_{3}=\frac{\Delta y}{\Delta x}-2 \pi i \eta^{+} \Delta y \\
c_{1}=\left[\frac{\Delta y}{\Delta x}\right]^{2}+2 \pi i \eta^{+} \frac{\Delta y^{2}}{\Delta x} \\
c_{4}=\frac{\Delta y}{\Delta x}+2 \pi i \eta^{+} \Delta y \\
f_{1}=-\left(2 \pi \eta^{+} \Delta y\right)^{2}-2-2\left[\frac{\Delta y}{\Delta x}\right]^{2}+2\left[\frac{\Delta y \eta}{\Delta t}\right]^{2} \\
f_{2}=-\left(2 \pi \eta^{+} \Delta y\right)^{2}-2-2\left[\frac{\Delta y}{\Delta x}\right]^{2}+2\left[\frac{\Delta y \bar{n}}{\Delta t}\right]^{2}
\end{gathered}
$$




$$
f_{3}=-\left(2 \pi \eta^{+}\right)^{2} \frac{\Delta x \Delta y}{2}-\frac{\Delta x}{\Delta y}-\frac{\Delta y}{\Delta x}+(\eta \Delta t)^{2} \Delta x \Delta y
$$

and $\bar{n}=\eta n(x, y)$. Finally

$$
\begin{gathered}
\mathrm{g}_{3}=\frac{-\eta^{2} \Delta \mathrm{x}+\mathrm{k}_{1} \frac{\Delta \mathrm{t}}{\omega}}{\eta^{2} \Delta \mathrm{x}+\mathrm{k}_{1} \frac{\Delta \mathrm{t}}{\omega}} \\
\mathrm{h}_{3}=-2 \mathrm{k}_{1} \mathrm{e}^{\mathrm{i}\left(\mathrm{k}_{1}+2 \pi \eta^{+}\right) \times} \frac{1}{\pi}\{\cos \pi(\mathrm{y}+\Delta \mathrm{y})-\cos \pi(\mathrm{y}-\Delta \mathrm{y})\}\left\{\mathrm{e}^{-\mathrm{i} \omega(\mathrm{t}+\Delta \mathrm{t})}-\mathrm{e}^{-\mathrm{i} \omega(\mathrm{t}-\Delta \mathrm{t})}\right\} \frac{1}{\omega} \frac{2 \Delta \mathrm{t}}{\Delta \mathrm{y}\left[\eta^{2} \Delta \mathrm{x}+\mathrm{k}_{1} \frac{\Delta \mathrm{t}}{\omega}\right]}
\end{gathered}
$$

The stability requirements are the same as in [1] and translate here into

$$
\Delta \mathrm{t} \leq \max _{\bar{\Omega}} \mathrm{n}(\mathrm{x}, \mathrm{y}) \frac{\Delta \mathrm{y}}{\left[1+\left[\frac{\Delta \mathrm{y}}{\Delta \mathrm{x}}\right]^{2}+\pi \frac{\pi \Delta \mathrm{y}^{2}}{\Delta \mathrm{x}} \eta^{+}+\left(\pi \Delta \mathrm{y} \eta^{+}\right)^{2}\right]^{\frac{1}{2}}}
$$

With the above scheme, the numerical procedure starts from a state of rest and is driven to time harmonic steady state as described in Figure 1.3.

\section{Numerical Results}

In this section we show some results to demonstrate the effectiveness of the method described earlier. In numerical computations, the choice of $\eta$ and $\eta^{+}$is crucial. If $\eta^{+}=0$, there will be no envelope transformation, and the solution will be sought with the usual finite difference approach. Such choices were mentioned in Section 3. Here, we reinforce this through one example, and the rest of the choices follow through the same recipe. In equation (3.12) for the one-dimensional situation, we had

$$
\eta^{*}=\mathrm{k} \max _{\mathrm{a}<\mathrm{x} \leq \mathrm{L}} \mathrm{n}(\mathrm{x})=2 \pi \eta^{+} .
$$

The two-dimensional an alogue of (5.1) applied to the wave guide problem $\left(P_{E}\right)$ is

$$
\eta^{*}=\mathrm{k}_{1} \max _{(\mathrm{x}, \mathrm{y}) \varepsilon \bar{\Omega}} \mathrm{n}(\mathrm{x}, \mathrm{y})=2 \pi \eta^{+}
$$

For instance, for the case of one mode propagating in the guide, we know that the frequency $k$ of the incident field must be in the range $(\pi, 2 \pi)$. If we consider $k=\frac{3 \pi}{2}$, then

$$
\mathrm{k}_{1}=\sqrt{\mathrm{k}^{2}-\pi^{2}}=\frac{\sqrt{5}}{2} \pi
$$


Moreover, if the maximum value of $n(x, y)$ is 1 in $\bar{\Omega}$, then $\eta^{+}=\frac{\sqrt{5}}{4}$. This procedure is then carricd out for higher order mode propagation and other frequencies. One must realize this choice is approximate; it does not hold exactly even in one dimension unless the index of refraction is a constant.

Our goal in these calculations is to obtain an efficient procedure that will yield results within a reasonable time and effort, but with an acceptable range of error. In fact, the code was tested on a Masscomp minicomputer, Zenith ZF-151 personal computer, and an IBM AT. Results are obtainable in under ten minutes of execution time in all cases although the Masscomp and IBM AT produce results two to three times faster than the Zenith. Without the wave envelope technique, about sixty grid points in the $\mathrm{x}$-direction were needed to obtain satisfactory results. Comparable results with the wave envelope technique required only 13 points in the axial direction. We compare our results with the results of Kreigsmann [4] using the same format.

Our first result is a trivial case where the index of refraction of the media $n(x, y) \equiv 1$. In this case, the wave form should be the same in the axial direction at every point from the inflow boundary to the outflow boundary. The value of $L$ was chosen to be 3 , so that $\bar{\Omega}=[-3,3] \times[0,1]$. The results are presented in Figure 5.1. In this figure the numbers printed at the mesh points are ten times the total field. Thus, the high values in the integers correspond to high intensity and the low values to low intensity of the electric field. Since our calculations yield $p(x, y, t)$, we used our tran sformation

$$
p(x, y, t)=e^{i 2 \pi \eta^{+}} v(x, y, t)=e^{i 2 \pi \eta^{+}-i k t} u(x, y)
$$

and calculated the absolute value $p$ to obtain $u$. Thus, the results are given for $u$. The frequency of oscillations is $k=\frac{3 \pi}{2}$. Also, reflection and transmission coefficients at the inflow and the outflow boundaries for values of $y=.3, .5$, and .7 are presented. They give a guideline for monitoring the accuracy of the solutions. As we see, the error in the calculation of the reflection coefficients is less than $1 \%$ and of the transm ission coefficients $4.5 \%$.

The next case we considered is that of a dielectric block imbedded in the guide with index of refraction $\frac{1}{\sqrt{3}}$, i.e., $m u(x, y)=\frac{1}{\sqrt{3}}$ for $(x, y), R=[-1,1] \times[0,1]$. Outside, (in $\left.R_{c}\right)$, the index of refraction is 1 . The steady harmonic state results reported in Figure 1.3 correspond to this situation. 
The time histories were constructed at the midpoint of the wave guide. Again, $\bar{\Omega}$ was chosen to be $[-3,3] \times[0,1]$, and the frequency $k$ is again $\frac{3 \pi}{2}$. The value of $\eta^{+}$is 0.559 . The results are given in Figure 5.2. Also the corresponding reflection and transmission coefficients are given. The exact solution can be obtained in this situation as pointed out in [4]. The error is almost 3\%, and they differ by at most $2.5 \%$ from the results of [4] with second-order boundary conditions.

Finally, the most interesting case of higher-order modes is presented: the value of $k$ is $\frac{5 \pi}{2}$, which propagates two modes. A dielectric block with $n^{2}=\frac{13}{9}$ is placed in the region $\mathrm{R}=\{(\mathrm{x}, \mathrm{y}) \quad 1-1 \leq \mathrm{x} \leq 1,0.2 \leq \mathrm{y} \leq 0.8\}$ For this case, we do not have exact results or comparisons, and computations are presented in Figure 5.3. 


\section{References}

[1] Baumeister, K. J., "Time-dependent wave envelope finite difference analysis of sound propagation," NASA TM-83744 (1984).

[2] Baumeister, K. J., "Finite difference theory for sound propagation in a lined duct with uniform flow using the wave envelope concept," NASA TP-1001 (1977).

[3] Hariharan, S. I. and R. C. MacCamy, "Integral equation procedures for eddy current problem s, " $J$. Comp. Phys., Vol. 45, No. 1 (1982), pp. 80-99.

[4] Kreigsmann, G. A., "Radiation conditions for wave guide problems," SIAM J. Sci. Statist. Comput., Vol. 3, No. 3 (1982), pp. 318-326.

[5] Morawetz, C. S., "The limiting amplitude principle," Comm. Pure Appl. Math., Vol. 15 (1962), pp. 181-197. 


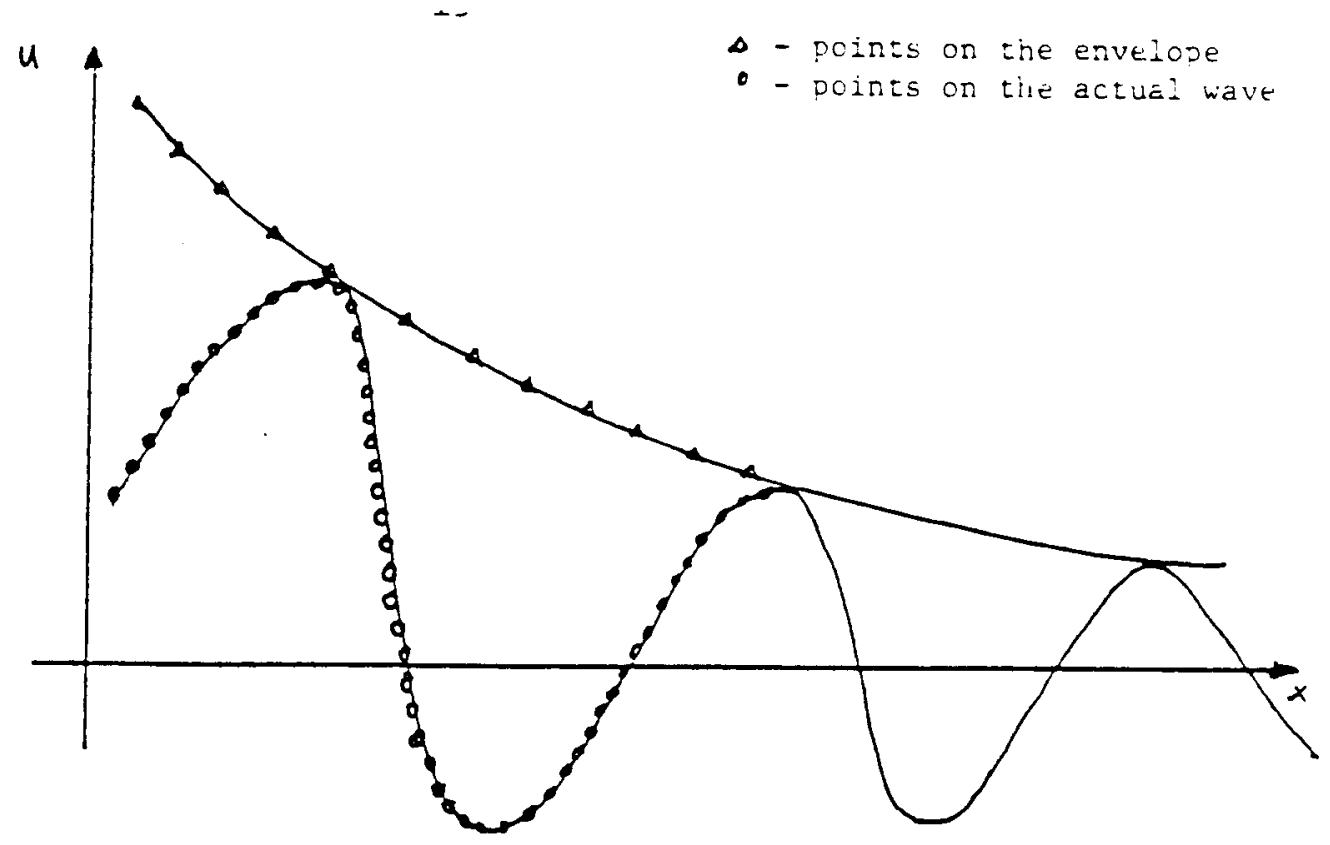

Figure 1.1

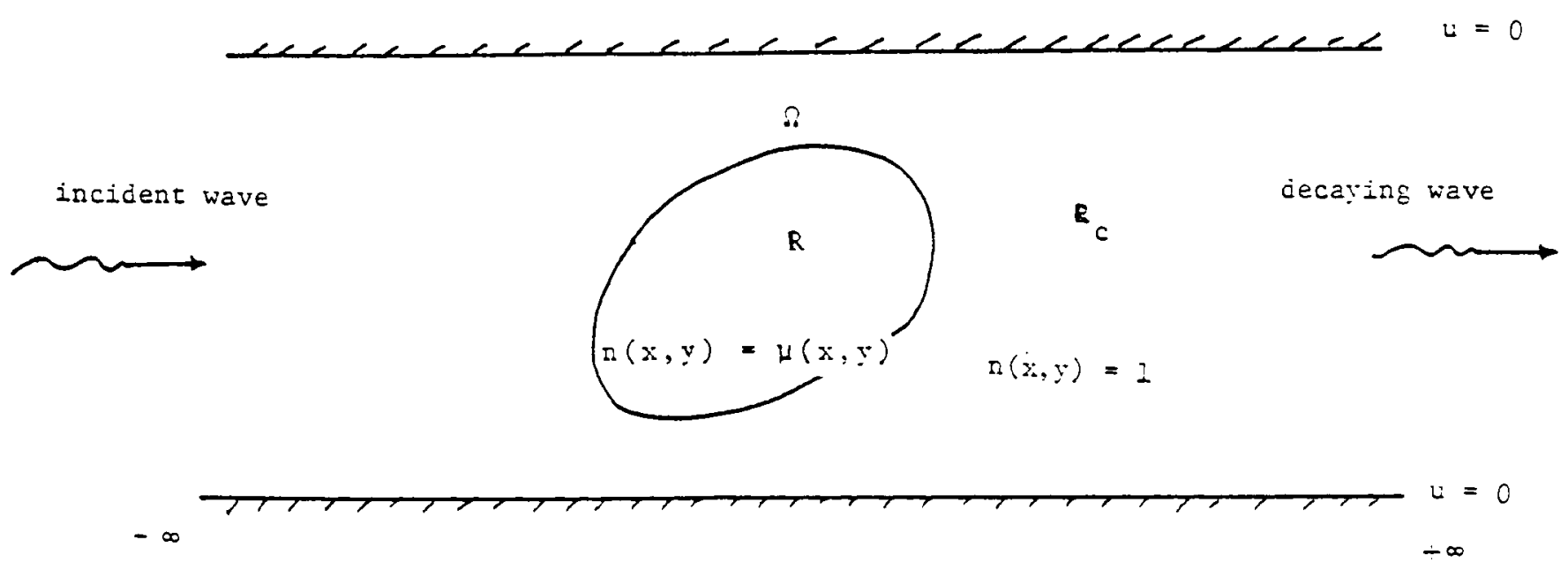

Pigure 1.2 
DF POOF:
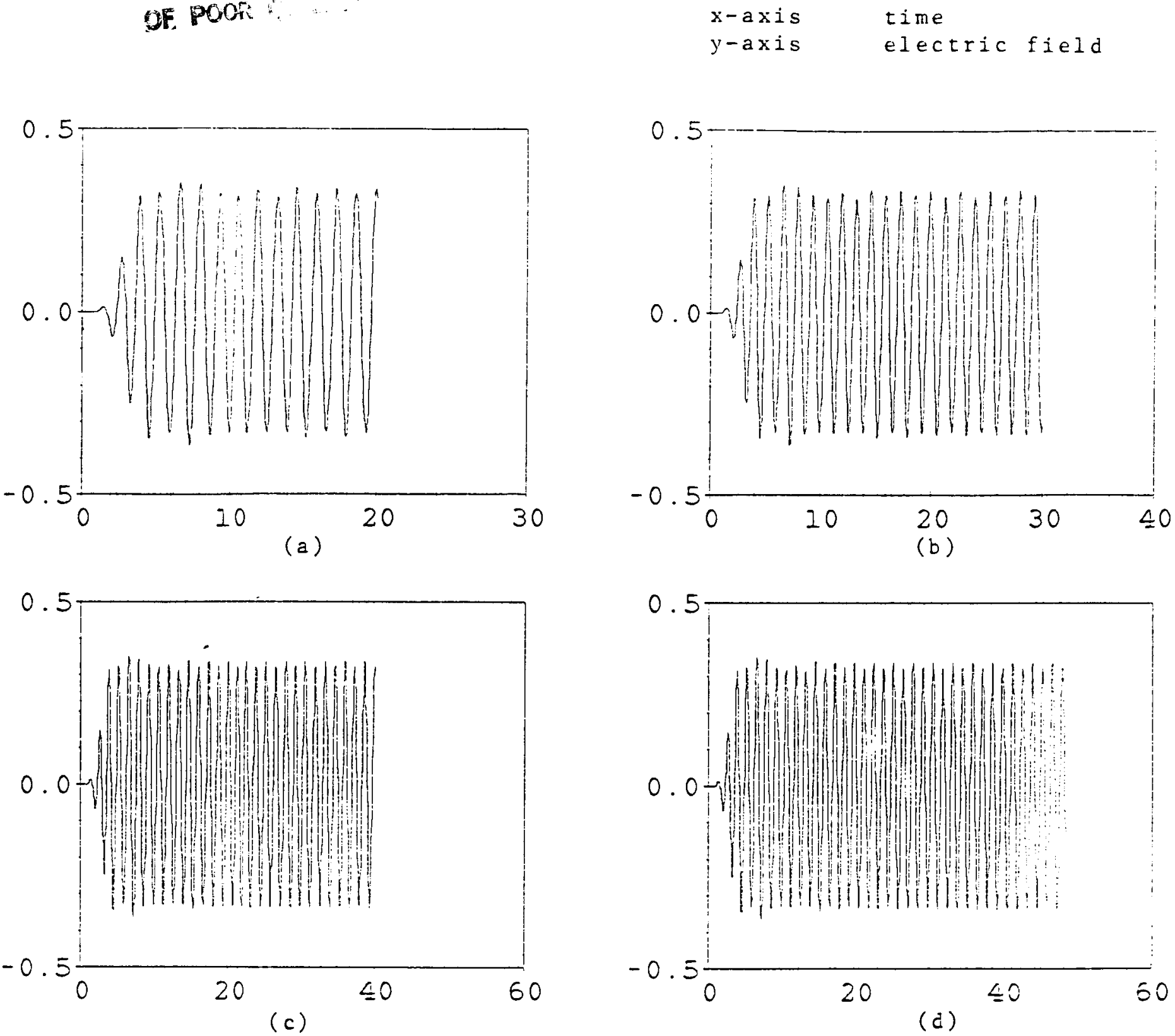

Figure 1.3 


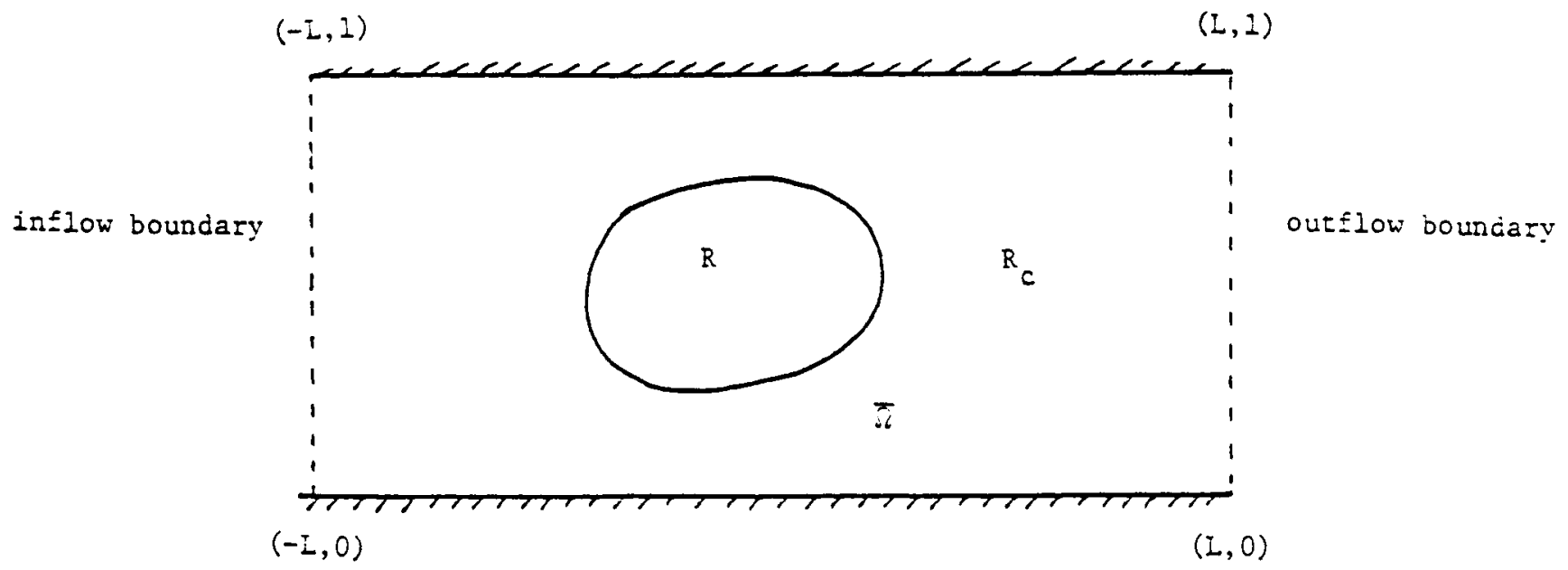

Figure 1.4

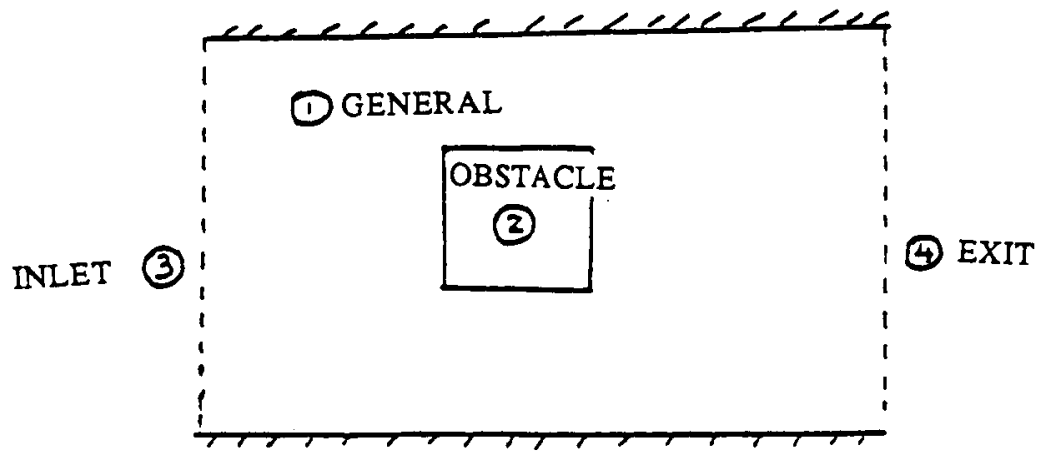

Pigure 4.1 


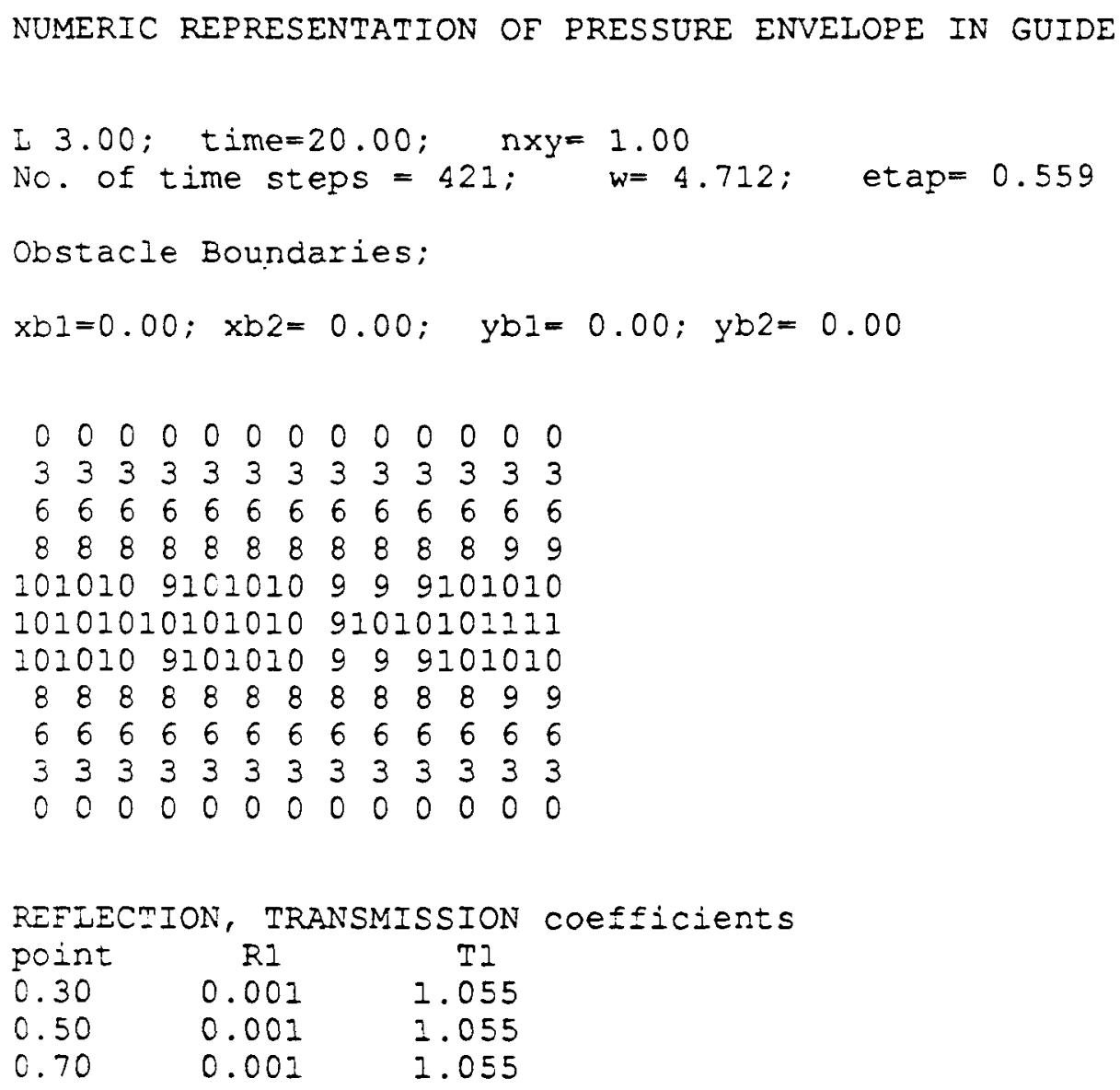

Figure 5.1. Numeric Representation of Pressure Envelope in Guide 


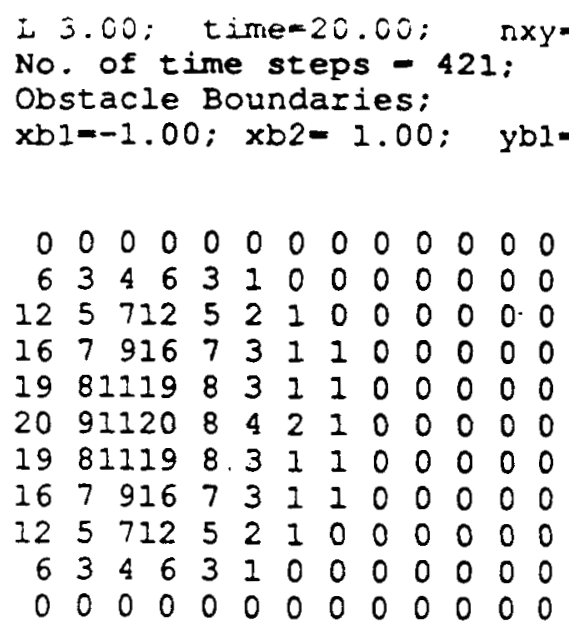

$\begin{array}{lcc}\text { REFIECTION, } & \text { TRANSMISSION } \\ \text { POInt } & \text { RI } & \text { TI } \\ \text { POEficients } \\ 0.30 & 1.006 & 0.024 \\ 0.50 & 1.006 & 0.024 \\ 0.70 & 1.006 & 0.024\end{array}$

Figure 5.2

NUMERIC REPRESENTATION OF PRESSURE ENVELORE IN GUIDE

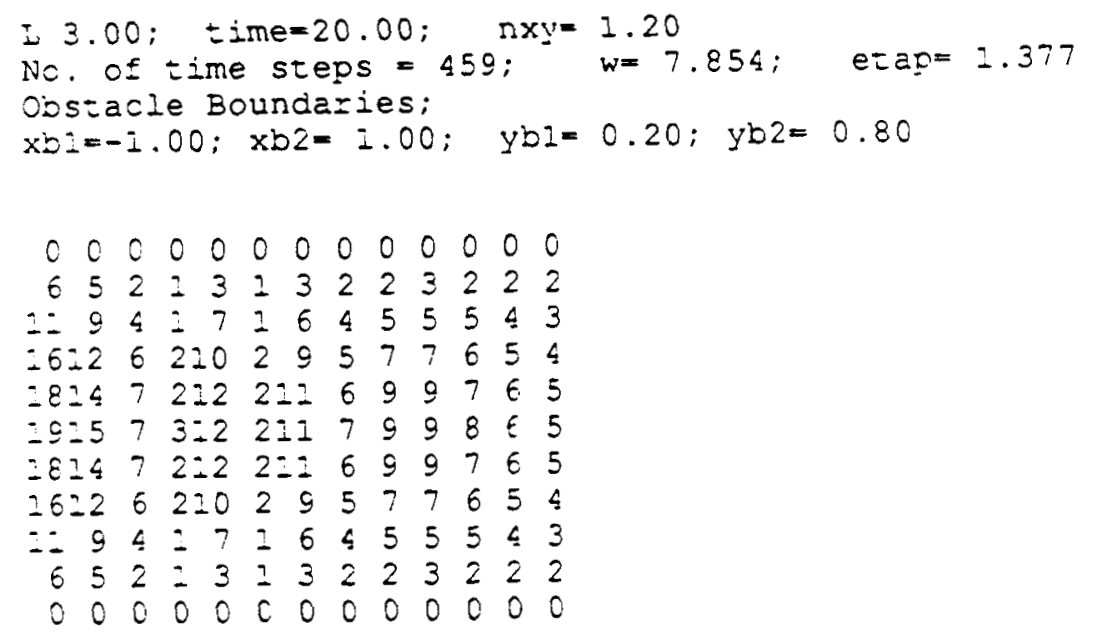


Standard Bibliographic Page

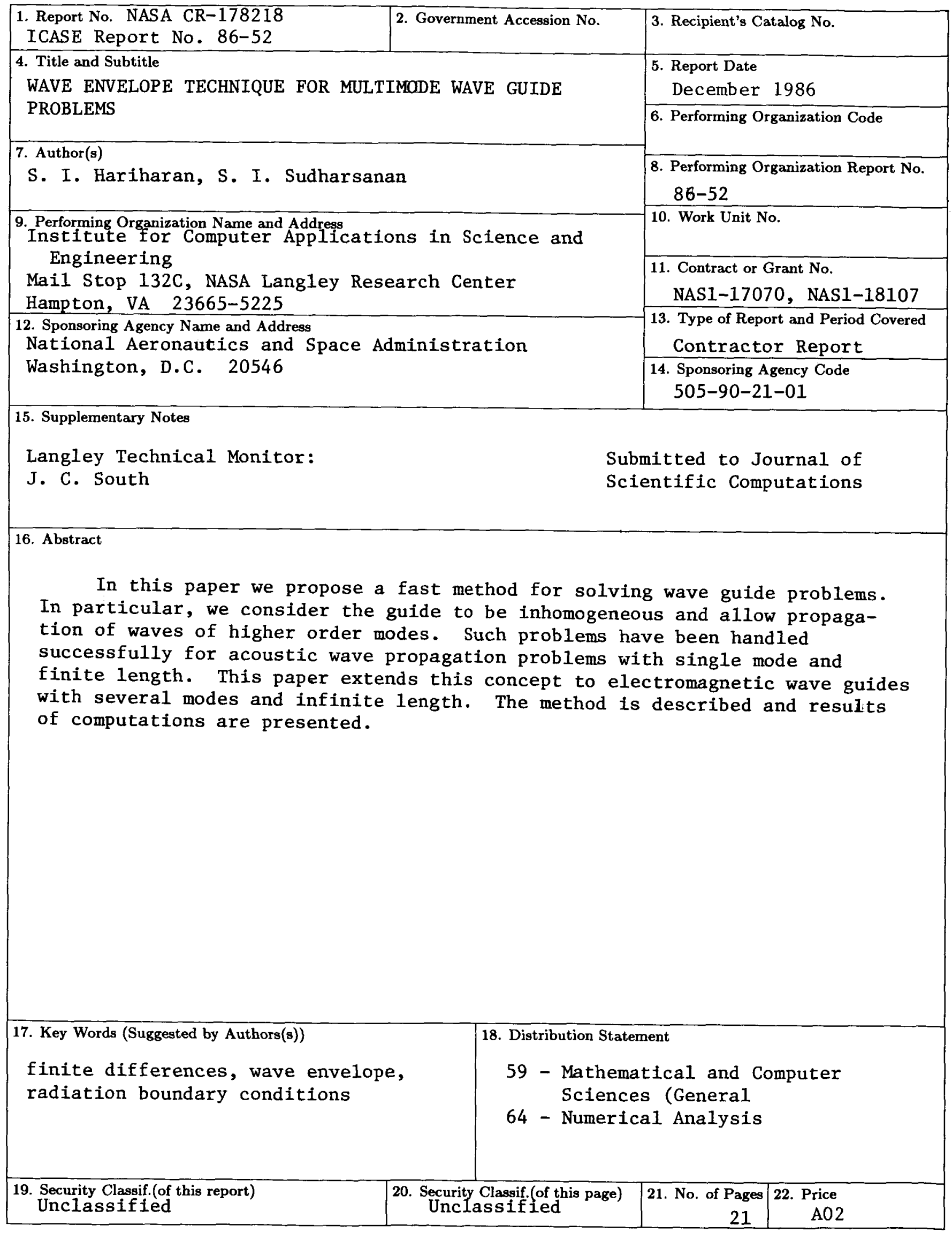

